\title{
Exploitations cacaoyères et pratiques culturales au sein d'un espace domanial : cas de la forêt classée du Haut-Sassandra (FCHS), Côte d'Ivoire
}

\author{
TIMITE Nakouana ${ }^{1,2}$, SANGNE Yao Charles $^{1,2}$, KPANGUI Kouassi Bruno ${ }^{1,2}$, BARIMA Yao \\ Sadaiou Sabas ${ }^{1,2}$ \\ ${ }^{1}$ Université JEAN LOROUGNON GUEDE - UFR Environnement - Côte d'Ivoire - BP 150 Daloa. \\ ${ }^{2}$ Groupe de Recherche Interdisciplinaire en Ecologie du Paysage et en Environnement (GRIEPE) - BP 150 Daloa (Côte \\ d'Ivoire) - www.griepe.net \\ Corresponding author:TIMITE Nakouana, tinatimmite@gmail.com.
}

Mots clés : Forêt classée du Haut-Sassandra, Populations infiltrées, Plantations cacaoyères, Pratiques culturales.

Keywords : Haut-Sassandra classified forest, infiltrated populations, cocoa plantations, farming practices.

Publication date 30/09/2019, http://www.m.elewa.org/JAPS

\section{RÉSUMÉ}

La forêt classée du Haut-Sassandra (FCHS) était l'une des forêts classées la mieux conservée avant la crise politico-militaire en Côte d'Ivoire. Cependant, elle a été massivement infiltrée par de nombreuses populations pendant la décennie de crises qu'a connue la Côte d'Ivoire de 2002 à 2011. La présente étude a pour objectif d'identifier les caractéristiques sociodémographiques des populations infiltrées et les pratiques culturales utilisées lors de la mise en place des plantations cacaoyères au sein de la FCHS. Pour y arriver, des enquêtes ont été réalisées de décembre 2017 à janvier 2018 auprès de 82 personnes sur environ une centaine de cacaoculteurs infiltrés dont les parcelles sont contenues dans le bloc 25 de la FCHS. Les enquêtes ont été suivies par des observations directes dans ces cacaoyères. Les résultats ont montré que 95,1\% des cacaoculteurs infiltrés sont des ressortissants Burkinabè, résidant dans les villages et campements riverains du bloc 25 de la FCHS. Ils sont majoritairement des jeunes cacaoculteurs ayant de petites plantations, généralement moins de 6 ha. La mise en place des plantations a débuté pendant la crise et s'est poursuivie après la période de conflits de 2011. Ces plantations ont été installées après défriche de la forêt par semis direct des fèves en utilisant un matériel végétal non sélectionné. Les fréquences de désherbage et de traitement phytosanitaire sont de deux à trois passages par an. Par ailleurs, la non maitrise des bonnes pratiques culturales cacaoyères, associée au manque d'encadrement (du fait de leur présence illégale au sein du domaine protégé de la FCHS), affecte le rendement annuel des plantations cacaoyères qui est d'environ $154 \mathrm{~kg} / \mathrm{ha}$ contre $395 \mathrm{~kg} / \mathrm{ha}$ au niveau national. 
Cocoa farms and farming practices within a federal area: the case of the classified forest of Haut-Sassandra (FCHS) (Côte d'Ivoire)

\section{SUMMARY}

The Haut-Sassandra classified forest (FCHS) was one of the best preserved classified forests before the politico-military crisis in Côte d'Ivoire. However, it was massively infiltrated by many populations during the decade of crises in Côte d'Ivoire from 2002 to 2011. The objective of this study is to identify the socio-demographic characteristics of the infiltrated populations and the farming practices used when setting up cocoa plantations within the FCHS. To achieve this, surveys were conducted from December 2017 to January 2018 among 82 people out of approximately 100 infiltrated cocoa farmers whose plots are contained in FCHS Block 25. The surveys were followed by direct observations in these cocoa farms. The results showed that $\mathbf{9 5 . 1 \%}$ of infiltrated cocoa farmers are Burkinabe nationals, residing in villages and camps along the banks of FCHS Block 25 . They are mainly young cocoa farmers with small plantations, generally less than 6 ha. The establishment of plantations began during the crisis and continued after the 2011 conflict period. These plantations were installed after clearing the forest by direct seeding of the beans using unselected plant material. The frequency of weeding and phytosanitary treatment is two to three times a year. In addition, the lack of good cocoa farming practices, combined with the lack of supervision (due to their illegal presence within the FCHS protected area), affects the annual yield of cocoa plantations, which is around $154 \mathrm{~kg} / \mathrm{ha}$ compared to $395 \mathrm{~kg} / \mathrm{ha}$ at national level.

\section{INTRODUCTION}

La problématique de la conservation de la forêt ivoirienne et de ses ressources est au centre des préoccupations des pouvoirs publics depuis les années 1960 (Nakouma et al., 2013). La superficie forestière ivoirienne estimée à 16 millions d'hectares en 1900 (Chatelain et al., 2004), ne représentait que 2,80 millions d'hectares en 2007 (FAO, 2007). Cette régression des superficies forestières s'est surtout accentuée pendant la décennie de crises qu'a vécue le pays. En effet, au cours de cette période, les domaines protégés ont connu une occupation illégale et anarchique qui a eu pour conséquence la dégradation des paysages forestiers (Kouakou et al., 2015 ; Assalé et al., 2016). Ces espaces protégés ont été fortement dégradés au profit d'activités agricoles, d'exploitation illicite du bois, de prélèvements incontrôlés des produits forestiers non ligneux, etc. C'est le cas de la forêt classée du HautSassandra (FCHS), qui était l'un des vestiges de la forêt dense semi-décidue et la mieux protégée avant les conflits en Côte d'Ivoire (Oszwald, 2005). La propension des populations pour la cacaoculture dans cet espace domanial y a suscité un afflux important de ces dernières (Zanh et al., 2016 ; Kouakou et al., 2017). Ainsi, près de $70 \%$ de la superficie de ce bloc forestier a été remplacé par des plantations cacaoyères. La mise en place de ces dernières est précédée par un abattage presque systématique de toutes les espèces arborescentes (Barima et al., 2016 a ; Barima et al., 2016 b ; Kouakou et al., 2017). Bien que la présence de ces populations au sein de cet espace domanial soit illégale, nul n'ignore la plus-value qu'occasionne leur production sur le volume de tonnage de cacao enregistré chaque année par la région. Cependant, les rapports sociologiques, l'origine des populations ayant infiltré la FCHS et les différentes pratiques culturales adoptées par ces dernières lors de la mise en place de leurs plantations cacaoyères sont très peu documentés. Pourtant, ces données sont importantes pour les décideurs et gestionnaires dans l'élaboration d'actions stratégiques et participatives à mettre en œuvre en vue de la reconstitution et de la préservation 
de la biodiversité de ce patrimoine. Dans ce contexte, la présente étude s'est donné pour objectif d'identifier les caractéristiques sociodémographiques des populations infiltrées et les différentes pratiques culturales utilisées lors de la mise en place des plantations au sein de la FCHS. De manière spécifique, il s'agira de

\section{METHODOLOGIE}

3.1 Milieu d'étude : La présente étude a été conduite dans la Forêt Classée du HautSassandra (FCHS) située à environ $60 \mathrm{~km}$ de la ville de Daloa entre $6^{\circ} 51^{\prime}$ et $7^{\circ} 24^{\prime}$ de latitude Nord, et $6^{\circ} 59^{\prime}$ et $7^{\circ} 10^{\prime}$ de longitude Ouest. Elle couvre une superficie de 102400 ha (SODEFOR, 1994). La FCHS est composée de plusieurs blocs forestiers, seul le bloc 25 (2319 ha) a fait l'objet de cette étude (Figure 1). Le bloc 25 est situé au Sud de la FCHS, à proximité de trois grands campements. Il s'agit des campements de Djarabanan, Amanikouadiokro et N'Doliyaokro. Le choix de ce bloc forestier a été motivé par la présence de quelques lambeaux de forêts plus ou moins dégradés encore disponibles, sa facilité d'accès et surtout la collaboration de la plupart des populations infiltrées avec la structure en charge de la surveillance de la forêt (la
: (1) Déterminer les caractéristiques sociodémographiques des cacaoculteurs clandestins installés dans la FCHS ; (2) Déterminer les caractéristiques agronomiques des plantations cacaoyères au sein de la FCHS ; (3) Identifier les pratiques culturales adoptées par les clandestins au sein de la FCHS.

SODEFOR). La zone est caractérisée par un climat de type tropical humide avec une pluviométrie oscillant entre 1200 et 1600 millimètres par an. La température moyenne annuelle est estimée à $26^{\circ} \mathrm{C}$ (SODEXAM, 2010). La végétation est composée par une plaine dont l'altitude moyenne est de $302 \mathrm{~m}$, toute la partie centrale de la zone est parsemée, d'Est en Ouest, de nombreux affleurements granitiques dont le plus élevé culmine à $449 \mathrm{~m}$ (Perraud, 1971). Aujourd'hui, du fait des actions anthropiques, la végétation qui était constituée d'une forêt dense humide semi-décidue (Guillaumet et Adjanohoun, 1971) a presque disparu laissant place à d'autres types de végétation tels que des forêts dégradées, des jachères et des plantations agricoles essentiellement les cultures vivrières et cacaoyères (Kouakou et al., 2015).
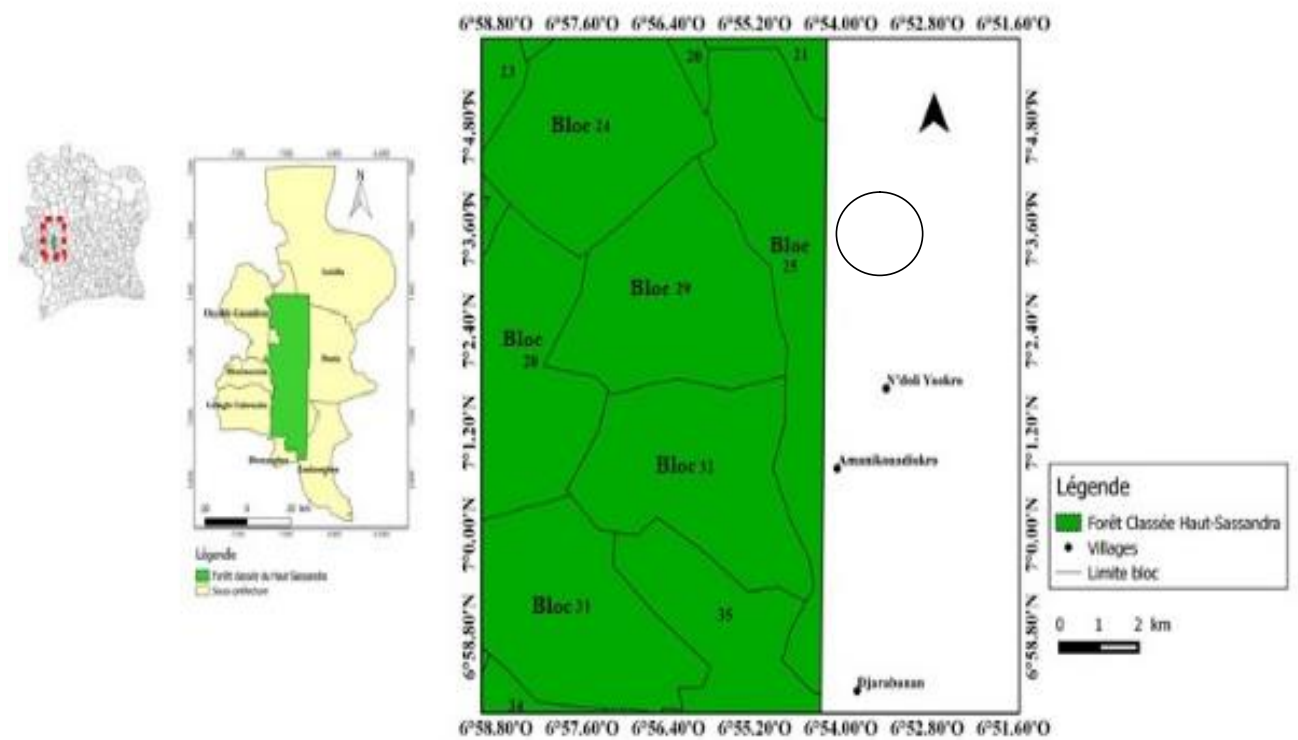

Figure 1 : Site d'étude 
3.2 Plan d'échantillonnage : La collecte des données de la présente étude a été effectuée auprès des cacaoculteurs clandestins de la FCHS. Le choix a été porté sur les cacaoculteurs car le cacao constitue la principale spéculation de ces populations infiltrées. Pour connaitre le profil des producteurs et des plantations cacaoyères au sein de la FCHS, nous avons procédé par une enquête à partir de questionnaire semi-structuré. L'enquête a été menée par une étudiante dans le cadre de ses travaux de recherches de décembre 2017 à janvier 2018. Les producteurs ont été interrogés individuellement dans leur plantation puis une observation des exploitations cacaoyères de chaque enquêté a été faite afin de confirmer les informations données. Au total 82 cacaoculteurs clandestins ayant coopérés ont été interrogés. L'enquête a permis de récolter les informations quantitatives et qualitatives sur ces personnes et leurs plantations de cacao. Sur la base de ces données, les caractéristiques sociodémographiques (âge ; origine ; provenance ; période d'installation) ont été déterminées. De même, les informations sur les caractéristiques des plantations (mode d'acquisition, précédent cultural, âge ; superficie et rendement), ainsi que les pratiques culturales adoptées (matériel végétal planté,

\section{RESULTATS}

\subsection{Caractéristiques}

sociodémographiques des cacaoculteurs clandestins de la FCHS : Les clandestins du bloc 25 de la FCHS sont constitués de Burkinabès, Maliens et Ivoiriens mais les plus représentés sont les Burkinabès (95,1 \%). L'infiltration de la FCHS s'est faite en deux vagues. Ainsi, la plupart des cacaoculteurs clandestins $(82,9 \%)$ ont infiltré la FCHS pendant la période post crise contre seulement $17,1 \%$ d'infiltrés durant la période de crises de mode de plantation ; désherbage ; traitements insecticides et conservation d'espèces ligneuses) ont été renseignées.

3.3. Traitement et analyse des données : A l'issu de l'enquête, les données collectées ont été dépouillées à l'aide du logiciel Sphinx plus². Les tableaux croisés dynamiques d'Excel 2010 ont permis de ressortir les fréquences des réponses apportées sur le profil des clandestins et les pratiques culturales. Pour établir le profil des exploitations au sein de la forêt classée du Haut-Sassandra, une analyse factorielle de correspondance (AFC) couplée à une classification ascendante hiérarchique $(\mathrm{CAH})$ a été effectuée grâce au package FactoMineR du logiciel R. L'AFC a permis d'analyser les données et de les visualiser sous forme de nuages de points. Quant à la Classification Ascendante Hiérarchique $(\mathrm{CAH})$, elle a permis de regrouper les individus présentant des caractéristiques voisines. En fonction de leur affinité, différents groupes ont été obtenus. Les variables actives utilisées sont : Le lieu de provenance, la période d'infiltration, le mode d'acquisition des parcelles, la superficie des plantations, le mode de plantation et le rendement moyen des plantations. La méthode de comparaison des moyennes utilisée a été celle de Tukey, au seuil de probabilité de $5 \%$.

2002-2011. Les clandestins résident dans les localités à la périphérie de la FCHS à savoir $45,12 \%$ à Djarabana situé à $3 \mathrm{~km}$ de la FCHS et $34,15 \%$ à Belleville à environ $13 \mathrm{~km}$. C'est une population quasiment jeune, $67 \%$ d'entre eux appartiennent à la tranche d'âge de 25 à 35 ans. (Tableau 1). Selon les infiltrés, les raisons qui ont motivé l'occupation de la FCHS sont principalement le coût élevé des parcelles $(42,68$ $\%$ et la difficulté d'accès à des terres cultivables à la périphérie de la FCHS (31,70\%). 
Tableau 1 : Caractéristiques sociodémographiques des populations infiltrées dans la FCHS

\begin{tabular}{lccc}
\hline \multicolumn{1}{c}{ Période d'infiltration } & $\begin{array}{l}\text { Pendant crise (\%) } \\
\mathbf{p}=\mathbf{1 7 , 1}\end{array}$ & $\begin{array}{l}\text { Post-crise (\%) } \\
\mathbf{p}=\mathbf{8 2 , 9}\end{array}$ & $\begin{array}{c}\text { Total général (\%) } \\
\text { Modalités }\end{array}$ \\
\hline Localités & 0,00 & 1,45 & 1,22 \\
Amidou carrefour & 38,46 & 34,78 & 35,36 \\
Belleville & 30,76 & 47,82 & 45,12 \\
Djarabana & 30,77 & 7,24 & 10,97 \\
Campement FCHS & 0,00 & 4,35 & 3,65 \\
Monokozoï & 0,00 & 4,35 & 3,65 \\
N'Doliyaokro & & & \\
\hline Nationalités & 100 & 94,2 & 3,12 \\
Burkinabé & 0,00 & 4,34 & 1,22 \\
Ivoirienne & 0,00 & 1,45 & \\
Malienne & & & 67,07 \\
\hline Classes d'âge & 61,54 & 68,11 & 20,73 \\
[25;35[ & 38,46 & 17,39 & 12,19 \\
[35;+ [ & 0,00 & 14,49 & \\
$<25$ & & & \\
\hline
\end{tabular}

$\mathrm{p}=$ proportion

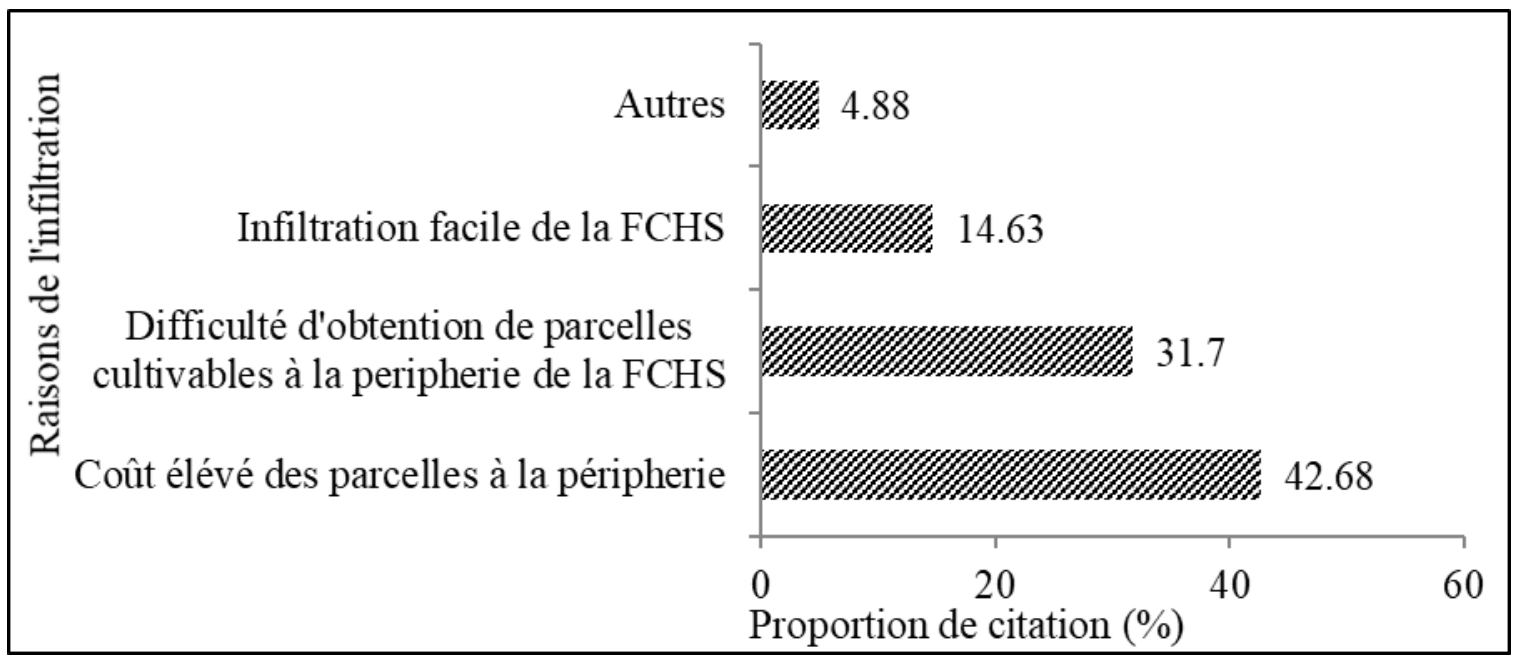

Figure 2 : Raisons évoquée justifiant l'infiltration dans la forêt classée du Haut-Sassandra

4.2 Caractérisation des plantations cacaoyères au sein de la FCHS

4.2.1 Mode d'acquisition des parcelles : Cinq modes d'acquisition des parcelles ont été identifiés au sein de la FCHS. Quelle que soit la période de l'infiltration, plus de la moitié des parcelles de forêt (59\%) ont été acquises par le paysan après son intrusion et $32 \%$ par métayage (travail partagé). Cependant, aucun mode d'acquisition des parcelles par achat, ni location, ni héritage n'a été identifié pendant la période de crise. 


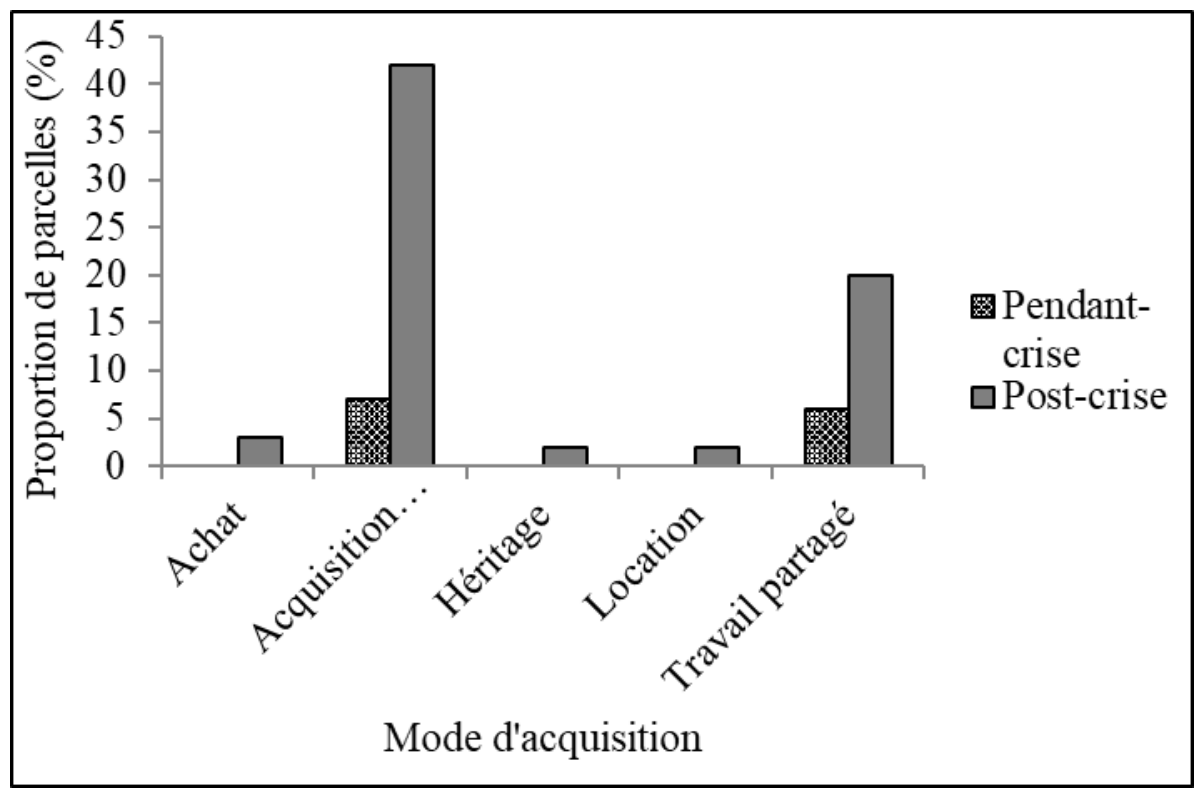

Figure 3 : Mode d'acquisition des parcelles en fonction de la période

4.2.2 Âge des plantations: L'âge des plantations dans la zone varie de 2 à 9 ans avec un âge moyen de 5,18 $\pm 1,17$ ans. Les plantations en début de production dont l'âge varie entre 4 et 7 ans ont été les plus dominantes (Tableau 2). L'analyse statistique de l'âge moyen des plantations en fonction de la période de l'installation a montré une différence hautement significative au seuil de $5 \%$ entre les différentes plantations (Tableau 3).

Tableau 2 : Quelques caractéristiques des plantations échantillonnées

\begin{tabular}{lll}
\hline Modalités & Classes & Proportion (\%) \\
\hline \multirow{2}{*}{ Age de la plantation (ans) } & $\leq 3$ & 13,41 \\
& {$[4 ; 7[$} & 70,73 \\
& $\geq 7$ & 15,85 \\
\hline \multirow{2}{*}{ Superficie de la plantation (ha) } & $\leq 2$ & 43,9 \\
& ] $2 ; 5]$ & 47,56 \\
& $\geq 6$ & 8,54 \\
\hline \multirow{2}{*}{ Rendement annuel de la plantation (kg/ha) } & $<100$ & 36,59 \\
& $\geq 100 ; 300[$ & 54,88 \\
\hline
\end{tabular}

4.23 Superficie des plantations : La superficie moyenne des plantations dans la zone est de 3,67 \pm 3,89 ha. L'analyse des données a montré que 43,9 \% des plantations ont une superficie inférieure à 2 ha et $47,56 \%$ ont une superficie comprise entre 2 et 5 ha. Le test de Tukey révèle une différence significative $(p<0,05)$ entre les superficies moyennes des plantations qui ont été installées pendant la crise (2002-2011) et celles installées en période post-crise ivoirienne c'est-à-dire après 2011 (Tableau 3). Les plantations installées pendant la crise ont les superficies moyennes les plus grandes $(7,30 \pm 7,96 \mathrm{ha})$.

4.2.4 Rendement des plantations : L'analyse des données relatives au rendement des plantations a montré que 54,88 \% des plantations échantillonnées ont un rendement 
compris entre 100 et $300 \mathrm{~kg} / \mathrm{ha}$ (Tableau 3). Le rendement moyen des plantations installées pendant la crise est de 206,92 $\pm 212,06 \mathrm{~kg} / \mathrm{ha}$ contre $141,32 \pm 106,46 \mathrm{~kg} / \mathrm{ha}$ pour celles installées en période post crise. Cependant le test statistique n'a montré aucune différence significative entre les rendements des plantations quelle que soit la période d'installation. Il est bon de noter que le rendement moyen des plantations dans la zone est de $151,72 \pm 129,45 \mathrm{~kg} / \mathrm{ha}$.

Tableau 3 : Caractéristiques des plantations par rapport à la crise

\section{Caractéristiques}

$$
\begin{array}{llll}
\text { Pendant-crise } & \text { Post-crise } & \text { Total général } & \operatorname{Pr}(>\mathrm{F})
\end{array}
$$

\begin{tabular}{lllll}
\hline Age moyen & $6,69 \pm 1,55$ & $4,89 \pm 1,14$ & $5,18 \pm 1,17$ & 0,000004 \\
Superficie moyenne & $7,30 \pm 7,96$ & $2,98 \pm 1,96$ & $3,67 \pm 3,89$ & 0,00014 \\
Rendement moyen & $206,92 \pm 212,06$ & $141,32 \pm 106,46$ & $151,72 \pm 129,45$ & 0,0939 \\
\hline
\end{tabular}

4.3 Pratiques culturales adoptées par les cacaoculteurs au sein de la FCHS

4.3.1 Matériel végétal planté et mode de mise en culture : Les plantations au sein de la FCHS ont été installées à partir de deux types de matériel végétal à savoir le matériel végétal ghana (Forasteros Haut-Amazonienne et hybrides) et le matériel végétal français (Amelonado). Par ailleurs certaines plantations ont été mises en place à partir du mélange de toutes les variétés (Tableau 4). Les plantations dont le matériel végétal est le Forasteros Haut Amazonienne et hybrides, représentent 54,87\% contre $36,58 \%$ pour celles issues du mélange de variétés et $8,54 \%$ pour celles créées essentiellement à partir de la variété Amelonado. Les paysans utilisent 3 procédés pour la mise en culture à savoir (le semis direct, les pépinières en pleine terre et les pépinières en sac plastique). Cependant, les plus utilisés parmi ces procédés sont la mise en pépinières en pleine terre $(47,56 \%)$ et le semis direct $(45,12 \%)$.

Tableau 4 : Variétés cultivées et mode de mise en culture

\begin{tabular}{lll}
\hline Modalités & Classes & Proportion (\%) \\
\hline \multirow{3}{*}{ Variétés culturales } & Amélonado & 8,54 \\
& Forasteros Haut-Amazonienne et hybrides & 54,87 \\
& Mélange de variétés culturales & 36,58 \\
\hline \multirow{3}{*}{ Mode de plantation } & Pépinières en pleine terre & 47,56 \\
& Pépinières en sac plastique & 7,32 \\
& Semis direct & 45,12 \\
\hline
\end{tabular}

4.3.2 Mode de création des plantations et entretien: Pour tous les paysans installés à l'intérieur de la FCHS, la plantation a été créée après défrichage du sous-bois forestier à la main à l'aide de machette. L'entretien des plantations a concerné le désherbage, les traitements à l'aide de pesticides. Les résultats ont mis en évidence que la totalité des paysans clandestins $(100 \%)$ pratiquent le désherbage manuel par l'usage de la machette pour assurer l'entretien de leurs plantations. La fréquence de désherbage est de 2 à 3 passages par an pour $56,09 \%$ des clandestins. En ce qui concerne les traitements phytosanitaires, ils concernent 
surtout les insecticides et sont pratiqués par plus de $95 \%$ des paysans contre seulement 4,88 $\%$ qui n'y ont pas recours. La fréquence des traitements phytosanitaires était de 2 à 3 traitements par an pour la plupart des paysans interrogés (Tableau 5).

Tableau 5 : Récapitulatif des pratiques adoptées pour la création et l'entretien des champs

\begin{tabular}{llc}
\hline Modalités & Classes & Proportion $\mathbf{( \% )}$ \\
\hline Défrichage & Manuel & 100 \\
Mode d'entretien & Produits phytosanitaires & 95,12 \\
\hline \multirow{2}{*}{ Traitement insecticide } & 1 & 2,44 \\
& 2 à 3 & 84,15 \\
& 4 et plus & 8,54 \\
\hline \multirow{2}{*}{ Désherbage } & 1 & 4,88 \\
& 2 à 3 & 56,09 \\
\hline
\end{tabular}

4.3.3 Pratique de l'agroforesterie : Sur les 82 plantations cacaoyères visitées au sein de la FCHS, L'association d'arbres a été observée dans $80,49 \%$ des plantations contre $19,51 \%$ de plantations qui sont conduits en plein soleil. L'inventaire floristique effectué a permis de recenser 87 espèces végétales reparties entre 71 genres et 31 familles. Les espèces les plus prépondérantes dans les plantations cacaoyères sont respectivement Celtis mildbraedii Engl. (17 individus), Napoleonaea vogelii (Hook.f.), Diospyros canaliculata (De Wild.) et Chidlowia sanguinea (Hoyle) avec chacune 11 individus. Baphia bancoensis (Aubrév) (10 individus), Corynanthepachyceras (K. Schum.) (9 individus), Celtis adolfi-fridericii Engl. (8 individus), Nesogordonia papaverifera (A. Chev.) (7 individus), Microdesmis keayana J. Léonard et Celtis zenkeri (Engl.) avec 6 individus chacune.

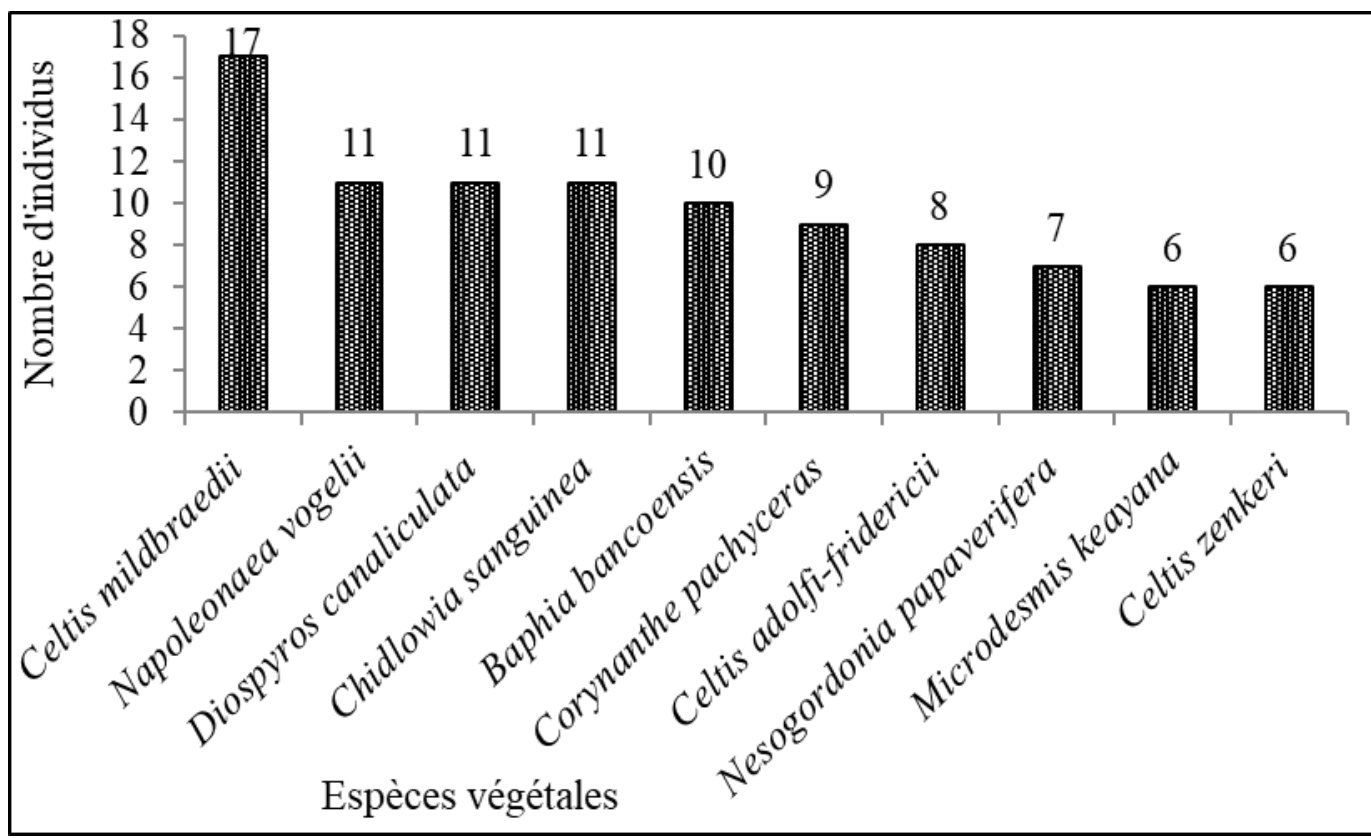

Figure 4 : Espèces prépondérantes laissées dans les plantations cacaoyères 
Les espèces conservées dans les plantations jouent de nombreux rôles. La plupart de ces espèces (43,94\%) servent d'ombrage, $(33,33 \%)$ servent de tuteurs aux ignames, $(12,12 \%)$ jouent le rôle de protection du sol contre l'érosion, $(7,58 \%)$ ont des vertus médicinales et $(3,03 \%)$ entrent dans l'alimentation (Figure 5).

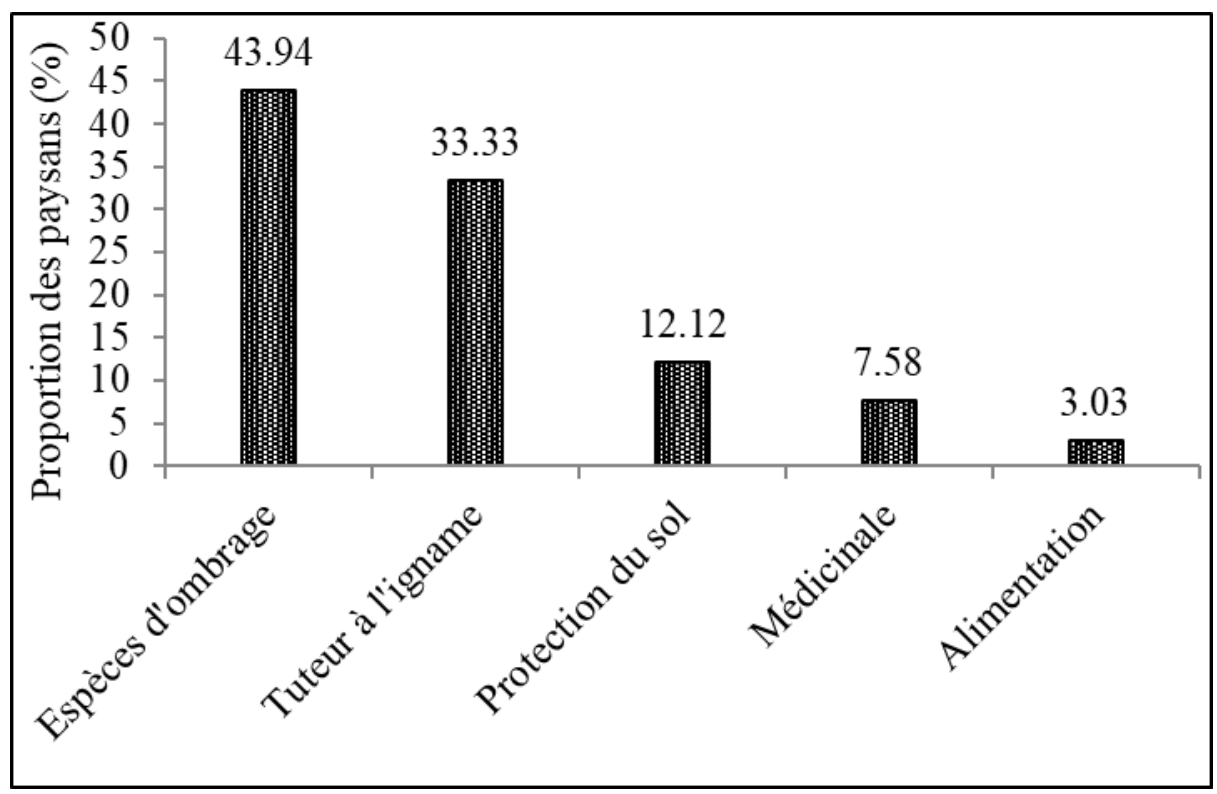

Figure 5 : Rôle des espèces conservées dans les plantations cacaoyères

\subsubsection{Profil des exploitations cacaoyères} au sein de la FCHS: Les différentes ordinations couplées à la classification ascendante hiérarchique des paysans enquêtés ont permis de discriminer trois groupes d'exploitations dans la forêt classée du HautSassandra (Figure 6) :

- $\quad$ Le premier groupe (G1) est caractérisé par les exploitations cacaoyères appartenant aux clandestins vivant dans les campements encore sis au sein de la FCHS. Ces clandestins ont infiltré la forêt pendant la période de crise en Côte d'Ivoire. Leurs plantations sont donc les plus âgées et les parcelles ont été majoritairement acquises par métayage Les exploitations de ce groupe ont des superficies de plus de 6 ha.

- $\quad$ Le deuxième groupe (G2) a rassemblé les exploitations cacaoyères dont les superficies sont comprises entre 1 et 5 ha. Elles ont été installées majoritairement dans la période postcrise par semis direct et pépinières en pleine terre. Le rendement annuel varie de 0 à $300 \mathrm{~kg}$ à l'hectare. Les clandestins propriétaires de ces exploitations sont majoritairement issus des localités de Djarabana; Belleville et N’Doliyaokro. Leursparcelles ont été acquises soit personnellement, soit par héritage.

- $\quad$ Le groupe 3 (G3) est composé des exploitations appartenant aux paysans infiltrés issus des localités de Monokozoï et Amidou Carrefour. Ces derniers ont acquis leurs parcelles soit par location, soit par achat. Les paysans de ce groupe procèdent par pépinières humides (usage de sachets plastiques) pour la mise en place de leurs exploitations cacaoyères. 


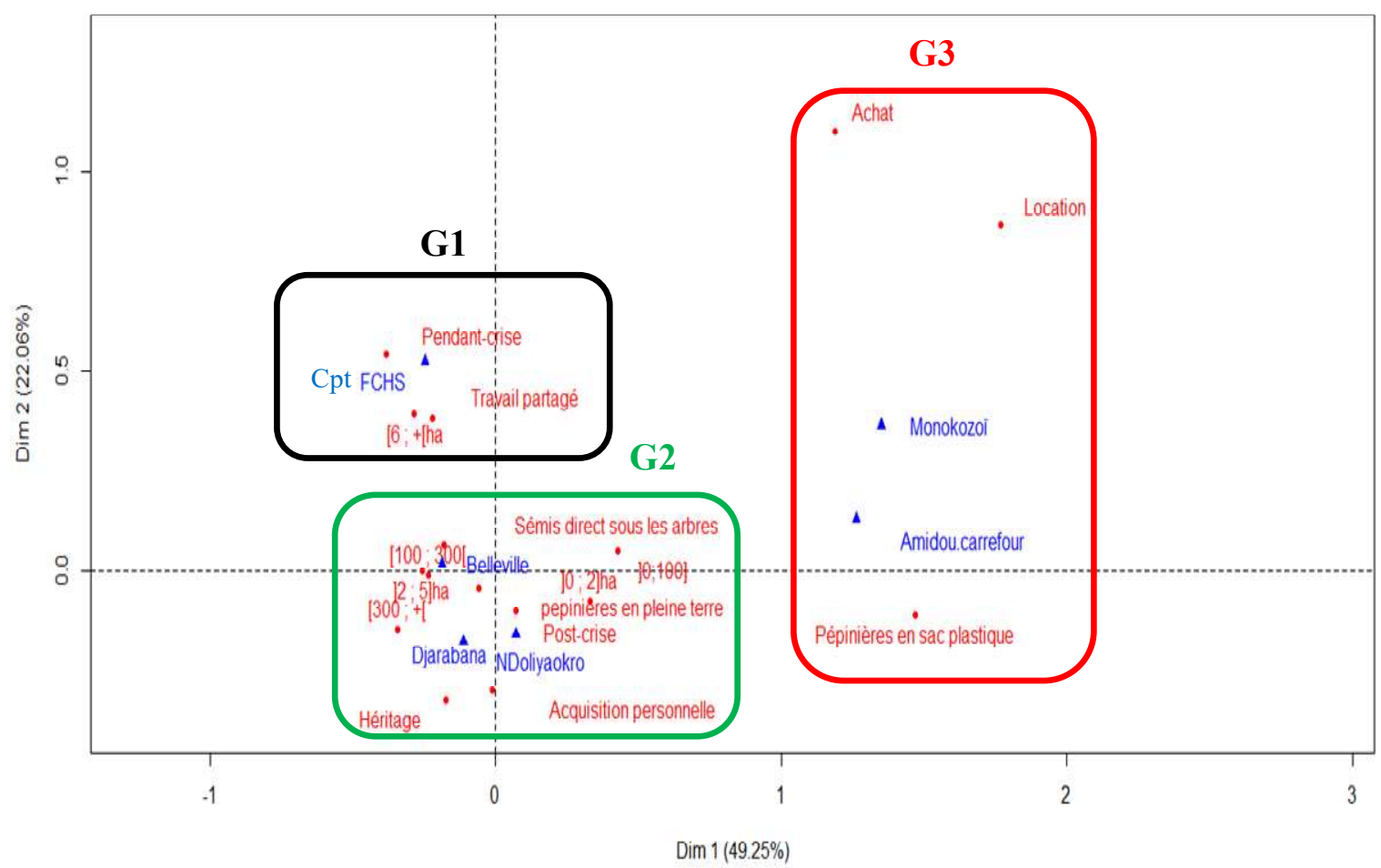

Figure 6 : Diagramme d'ordination des exploitations cacaoyères au sein du bloc 25 de la forêt classée du Haut-Sassandra

\section{$5 \quad$ DISCUSSION}

5.1 La majorité des infiltrés sont des allogènes, conséquence de la crise ivoirienne : Les personnes impliquées dans la cacaoculture au sein de la FCHS sont en majorité des allogènes, principalement des ressortissants du Burkina Faso (95,10\%). Ces populations sont non seulement les plus nombreuses mais aussi celles qui ont été les premières à infiltrer la FCHS pendant la période de crise de 2002 à 2011. Cette forte proportion de Burkinabés pourrait être imputable d'une part, à la situation de crise politico-militaire qu'a connue la Côte d'Ivoire de 2002 à 2011 caractérisée par l'absence des autorités en charge de la surveillance des domaines protégés et d'autre part à la saturation des terres cultivables dans le domaine rural. Ce dernier facteur a fortement contribué à une modification profonde dans le mécanisme de cession ou d'acquisition des terres (anciennement les terres étaient "données" moyennant une bouteille de liqueur ou après quelques années de travail au service d'un propriétaire terrien, etc.) au profit de la vente. Dès lors, les coûts déjà exorbitants des terres aggravés souvent par des rapports intercommunautaires dégradés du fait de la crise post-électorale (populations autochtones accusant les allogènes dont les ressortissants du Burkina Faso d'être les responsables de la crise), pourraient justifier dans une certaine mesure l'occupation de FCHS alors «sans propriétaire ». Au sein de la FCHS, leur activité principale est la cacaoculture ainsi que l'ont déjà signalée plusieurs travaux dont ceux de Sangne et al., 2015 ; Barima et al., 2016 ; Kouakou et al., 2016). La situation a été la même ailleurs dans d'autres forêts classées et aires protégées en Côte d'Ivoire comme ce fut dans les parcs nationaux de la Marahoué et du Mont Péko (Dibi et al., 2008; Gone $\mathrm{Bi}$ et al., 2013; Kouamé, 2016), dans les forêts classées de la Béki et la région du Cavally (Atta et al., 2017 ; RAIDH, 2017). Par ailleurs, la destruction 
rapide de la FCHS (102400 ha) en l'espace de 10 ans (soit l'équivalent de 14300 terrains de football par jour), est imputable non seulement au nombre de clandestins, mais surtout au fait qu'ils sont en majorité des jeunes (âge majoritairement compris entre 25 et 34 ans). Les résultats de l'analyse factorielle des correspondances ont permis de montrer que les premières populations installées dans la FCHS, l'ont infiltrée pendant la période de crise de 2002-2010. Elles ont ainsi acquis les plus grandes superficies (G1). Ces paysans ont ensuite attiré leurs confrères (compatriotes) résidant majoritairement dans les localités (villages et campements) riveraines : Belleville, Djarabana, Monokozoï et N'Doliyaokro vers la forêt. Ces derniers ont infiltré la FCHS vers la fin de la crise post-électorale de 2011. Une fois dans la FCHS, les derniers venus ont travaillé comme employé dans les plantations des premiers pour avoir leur portion de plantations d'où les superficies réduites des plantations cacaoyères de ceux-ci (G2 et G3).

5.2 L'adoption de mauvaises pratiques de la cacaoculture, source de mauvais rendement et de propagation de maladies : L'étude a révélé que la variété «Ghana » est la plus utilisée par les paysans lors de la mise en place de leur exploitation. Cela pourrait s'expliquer soit par la facilité d'obtention de cette variété dans la zone ou la difficulté d'accès à la variété améliorée par les paysans du fait de leur statut de clandestins. Signalons que cette variété «Ghana » est la plus sollicitée par les producteurs de cacao en Côte d'Ivoire. De ce fait, elle est répandue dans presque toutes les grandes régions productrices de cacao en Côte d'Ivoire (Assiri et al., 2009 ; Kpangui, 2015 ; Cissé et al., 2016). Toutefois, le semis direct des fèves signalé comme principale technique mise en œuvre par les clandestins s'expliquerait (selon les propos recueillis auprès des infiltrés) par le fait que le sol forestier de la FCHS etait encore très fertile. Il n'était donc pas nécessaire de procéder par des pépinières. Seules les dernières vagues de clandestins venues des villages de Monokozoï et Amidou Carrefour (G3) dans la FCHS ont eu recours à ces dernières techniques culturales. Par ailleurs, la présente étude a mis en évidence que les exploitations cacaoyères au sein de la FCHS ne sont pas régulièrement désherbées $(2$ à 3 fois/an), ni traitées aux produits phytosanitaires contre les attaques fongiques et autres mirides du cacaoyer. A cela, il faut ajouter un autre fait relatif à l'infestation de la plupart des champs par certaines maladies notamment le Swolen shoot, et par certains parasites végétaux tels que le gui (g. Tapinanthus). Il en résulte une baisse du rendement des champs estimés à $154 \mathrm{~kg} / \mathrm{ha}$ contre $395 \mathrm{~kg} / \mathrm{ha}$ au niveau national (Assiri et al., 2009). Toutes ces pratiques qui ne sont pas conformes aux bonnes pratiques culturales sont le fait de plusieurs raisons. En effet, du fait de leur présence illégale au sein de la FCHS (espace protégé de l'Etat), les clandestins sont obligés de vivre en fugitifs loin du regard des agents des Eaux et forêts. L'autre raison est surtout la méconnaissance-même des bonnes pratiques de la cacaoculture dû à un déficit d'encadrement, à l'absence de ressources nécessaires pour l'achat des produits agropharmaceutiques homologués dont les coûts oscillent entre 5500 et 7500 FCFA selon les paysans. Par ailleurs, le semis direct à partir de fèves prélevées directement sur des pieds dont les clandestins ignorent l'état de santé a vraisemblablement contribué à l'expansion de maladies notamment le Swollen shoot dans la plupart des exploitations de la FCHS. Tous ces facteurs pourraient justifier le faible rendement des plantations.

5.3 La forêt classée du Haut Sassandra, quel avenir ?: Lorsqu'on considère l'âge moyen des plantations cacaoyères dans la FCHS (5,18 ans), on note une forte proportion de jeunes plantations. Cela laisse supposer que malgré la fin de la crise en 2011 et le retour des agents de la Société de développement des forêts (sodefor), les défrichements se poursuivent toujours. En effet, les observations faites sur le terrain dans le cadre de la présente étude ont permis de mettre en évidence de nouveaux défrichements et la présence de plants de moins d'un an (-1 an). Cette situation a déjà été signalée par plusieurs travaux 
antérieurs (Kouakou et al., 2015 ; Sangne et al, 2015 ; Barima et al., 2016) dans cet espace domanial. On pourrait dire que la Sodefor, gestionnaire légal des forêts classées en Côte d'Ivoire, n'a plus la main mise sur la FCHS. L'insuffisance du matériel logistique déjà vétuste, mais surtout l'insuffisance-même des agents forestiers pourrait justifier cette situation. Par ailleurs, Assalé et al., 2016 a révélé en 3 étapes le processus de mise en place de leurs cacaoyères par les clandestins dont: le défrichement du sous-bois forestier suivi d'un semi direct des fèves de cacao sous l'ombrage des grands arbres, la mise à mort sur pied des arbres soit au feu, soit à l'aide de produits chimiques environ 6 à 18 mois plus tard, qui conduit enfin à la mort des arbres laissant apparaître la jeune plantation de cacaoyer. Au terme de ce processus, tous les arbres dans les champs doivent disparaitre pour ne laisser la place qu'aux cacaoyers. Cette pratique est contraire à celles adoptées généralement par les paysans installés dans le domaine rural à la

\section{CONCLUSION}

Les clandestins pratiquant la cacaoculture dans la FCHS sont principalement jeunes et d'origine burkinabé. Ils résident pour la plupart dans les villages environnant la FCHS. La quasi-totalité des exploitations cacaoyères $(82,9 \%$ ) du bloc 25 de la FCHS ont été mises en place vers la fin de la période de crise qu'a traversée le pays de 2002 à 2011. Ce sont de petites exploitations installées après défriche de la forêt. Les plantations ont été principalement mises en place à partir des variétés non sélectionnées par semis direct. Le déficit d'entretien des plantations, l'âge des plantations et l'utilisation

\section{REMERCIEMENTS}

La présente étude a été réalisée dans le cadre du projet RN-FCHS intitulée «Une reconstitution naturelle de la forêt classée du Haut-Sassandra après abandon des activités agricoles est-elle possible ? ». Ce projet a été initié conjointement périphérie de la FCHS. Ces derniers en effet, maintiennent toujours dans leurs plantations plusieurs espèces d'arbres locales dont plusieurs sont des semenciers (Terminalia superba, Irvingia gabunensis, Triplochiton scleroxylon, Entandrophragma $s p$ ) et plusieurs autres arbres fruitiers (Persea americana, Citrus aurantifolia, Citrus sinensis, Cola nitida). En conséquence, il est certain que les pratiques culturales utilisées par les clandestins au sein de la FCHS ne puissent pas favoriser dans le court ou moyen terme une reconstitution de cet espace protégé. Cette situation pourrait être exacerbée par les superficies de plus en plus faibles $(3,67$ ha en moyenne) des exploitations des clandestins du fait de la saturation foncière au sein-même de la FCHS. Car en effet, les éléments (la fertilité des sols, charge semencière du sol, microorganismes du sol, $\mathrm{pH}$ du sol, etc.) qui devraient présider à une reconstitution du paysage forestier seront plus affectés, du fait de la forte réduction du temps de mise en jachère voire l'absence de mise en jachère.

des variétés non sélectionnées sont à la base du faible rendement des vergers de cacaoyers dans cette zone. Afin de freiner la perte de la biodiversité au détriment des cultures, il est nécessaire d'associer les populations au processus de reboisement de la forêt tout en préconisant l'association d'un grand nombre d'essences ligneux dans les plantations cacaoyères. L'interdiction de nouvelles friches dans la FCHS aiderait à maintenir dans ce milieu un minimum d'écosystème forestier qui aidera plus tard à une reconstitution naturelle de cette forêt.

par la SODEFOR et l'Université Jean Lorougnon Guédé (UJLoG) et est financé par le programme Sud Expert plantes pour le Développement Durable (SEP2D). 


\section{BIBLIOGRAPHIQUE}

Assiri AA, Yoro GR, Deheuvels O, Kebe BI, Keli ZJ, Adiko A. et Assa A : 2009. Les caractéristiques agronomiques des vergers de cacaoyers (Theobroma cacao L.) en Côte d'Ivoire. Journal of Animal \& Plant Sciences, 2 (1) : 55-66.

Atta JMK, Robin M, Toure A, Pottier P. et Oswald J : 2017. Déforestation et conflits fonciers dans les forêts classées de Béki et de Bossématié dans l'est de la côte d'ivoire. In : Espaces et tensions en Afrique subsaharienne. Ed. Universitaires Européennes : 357-387.

Barima YSS, Kouakou ATM, Bamba I, Sangne CY, Godron M, Andrieu J. et Bogaert J : 2016 a. Cocoa crops are destroying the forest reserves of the classified forest of Haut-Sassandra (Ivory Coast). Global Ecology and Conservation, 8 : 85-98.

Barima YSS, Assalé AAY, Vignal M, Andrieu J.et Godron M : 2016 b. Caractérisation post conflits armés des perturbations dans la Forêt Classée du Haut-Sassandra en Côte d'Ivoire. Afrique SCIENCE, 12(6) : 66-82.

Abdoulaye C, Kouadio AJC, Djaha K, Aimé VBT. et Yves AYC: 2016. Caractérisation des pratiques agroforestieres à base de cacaoyers en Zone de forêt dense semi-décidue : cas de la localité de Lakota (Centre-ouest, Cote d'Ivoire). European Scientific Journal ESJ, 12(21) : 50-69.

Chatelain C, Dao QH, Gautier L. et Spichiger RE : 2004. Forest cover changes in Côte d'Ivoire and Upper Guinea. In : Bongers F., Loorens, P., Kouamé, F. N., Hawthorne, W. D (Ed.). Biodiversity of West African forests: an ecological atlas of woody plant species. Wallingford : CABI Publishing, Wallingford (UK), 2004. pp 45-82.

FAO : 2007. Situation des forêts en 2002. Rome. 143 p.

Guillaumet JL. et Adjanohoun E: 1971. La végétation de la Côte d'Ivoire.
Kouakou ATM, Barima YSS, Kouakou KA, Kouame NF, Bogaert J. et Kouadio YJ : 2015. Forest dynamics in the north of the classified forest of Haut-Sassandra during the period of armed conflicts in Ivory Coast. American Journal of Sciences, 3(5) : 375-382.

Kouakou ATM, Barima YSS, Konate S, Bamba I, Kouadio YJ. et Bogaert J: 2017. Gestion des forêts domaniales en période de conflits : cas de la forêt classée du Haut-Sassandra, CentreOuest de la Côte d'Ivoire. International Journal Biological. Chemical Sciences, 11(1) : 333-349.

Kouakou KA, Barima YSS.et Zanh GG : 2017. Inventaire et disponibilité des produits forestiers non-ligneux utilisés par les populations riveraines de la Forêt Classée du Haut-Sassandra après la période de conflits armés en Côte d'Ivoire. Tropicultura, 35 (2) : 121-136.

Kouakou KA, Barima YSS, Kouakou ATM, Sangne YC, Bamba I. et Kouame NF : 2015. Diversité végétale post-conflits armés de la forêt classée du HautSassandra (Centre-Ouest de la Côte d'Ivoire). Journal Animal Plant Sciences, 26(2) : 4058-4071.

Kouamé WK : 2016. Le parc national du Mont Péko (Côte d'Ivoire) entre dynamiques de déguerpissement, tensions sociales et logiques des acteurs : vers un risque d'explosion de violences. Etudes caribéennes: 33-34. http://journals.openedition.org/etudesc aribeennes/ 9418 ; DOI : 10.4000 / études caribéennes. 9418

Kpangui KB : 2015. Dynamique, diversité végétale et valeurs écologiques des agroforêts à base de cacaoyers de la Sous-préfecture de Kokumbo (Centre de la Côte d'Ivoire). Thèse de Doctorat. UFR Biosciences. Université Félix Houphouët-Boigny (Abidjan. Côte d'Ivoire). 227 p. 
Perraud A : 1971. Les sols in le milieu naturel de la Côte d'Ivoire. Mémoires ORSTOM, (50), 269-391.

RAIDH : 2017. Déforestation : défis environnementaux et humanitaires dans la région du Cavally. Rapport d'alerte. Côte d'Ivoire. $52 \mathrm{p}$.

Sako N, Beltrando G, Atta K, Dibi N'daH. et Brou $\mathrm{T}:$ 2013. Dynamique forestière et pression urbaine dans le Parc national du Banco (Abidjan, Côte d'Ivoire). [VertigO] La revue électronique en sciences de l'environnement, 13(2).

Sangne CY, Barima YSS, Bamba I. et N’Doumé CTA : 2015. Dynamique forestière postconflits armés de la Forêt Classée du Haut-Sassandra (Côte d'Ivoire). VertigO - la revue électronique en sciences de l'environnement. 15(3). 